\title{
Therapeutic effects of various methods of MSC transplantation on cerebral resuscitation following cardiac arrest in rats
}

\author{
KA-HONG LEONG ${ }^{1,2^{*}}$, LI-LI ZHOU ${ }^{1,2^{*}}$, QING-MING LIN ${ }^{3}$, \\ PENG WANG $^{1,2}$, LAN YAO ${ }^{1,2}$ and ZI-TONG HUANG ${ }^{1,2}$ \\ ${ }^{1}$ Emergency Department, Sun Yat-sen Memorial Hospital; ${ }^{2}$ Institute of Cardiopulmonary Cerebral Resuscitation, \\ Sun Yat-sen University, Guangzhou, Guangdong 510120; ${ }^{3}$ Emergency Department, Fujian Provincial Hospital, \\ Fujian Medical University, Fuzhou, Fujian 350001, P.R. China
}

Received January 30, 2015; Accepted November 25, 2015

DOI: $10.3892 / \mathrm{mmr} .2016 .4927$

\begin{abstract}
In the present study, mesenchymal stem cells (MSCs) were transplanted into the brain of rats following cardiopulmonary resuscitation (CPR) by three different methods: Direct stereotaxic injection into the lateral cerebral ventricle (LV), intra-carotid administration (A), and femoral venous infusion (V). The three different methods were compared by observing the effects of MSCs on neurological function following global cerebral hypoxia-ischemia, in order to determine the optimum method for MSC transplantation. MSCs were transplanted in groups $\mathrm{A}, \mathrm{V}$ and $\mathrm{LV}$ following the restoration of spontaneous circulation. Neurological deficit scale scores were higher in the transplantation groups, as compared with the control group. Neuronal damage, brain water content and serum levels of S100 calcium-binding protein B were reduced in the hippocampus and temporal cortex of the transplantation groups, as compared with the control rats following resuscitation. MSCs were able to migrate inside the brain tissue following transplantation, and were predominantly distributed in the hippocampus and temporal cortex where the neurons were vulnerable during global cerebral ischemia. These results suggest that transplantation of MSCs may notably improve neurological function following CPR in a rat model. Of the three different methods of MSC transplantation tested in the present study, LV induced the highest concentration of MSCs in brain areas vulnerable to global cerebral ischemia, and therefore, produced the best neurological outcome.
\end{abstract}

Correspondence to: Dr Zi-Tong Huang, Emergency Department, Sun Yat-sen Memorial Hospital, Sun Yat-sen University, 107 Yanjiang West Road, Guangzhou, Guangdong 510120, P.R. China

E-mail: huangzitong_zt@163.com

*Contributed equally

Key words: bone marrow mesenchymal stem cells, global cerebral ischemic injury, cerebral resuscitation, cardiac arrest, transplantation

\section{Introduction}

According to statistics from the World Health Organization, cardiovascular disease resulted in $\sim 17,100,000$ cases of mortality in 2004, accounting for $29 \%$ of total global mortality (1). This is projected to rise to $23,600,000$ by 2030 (1). Cardiac arrest (CA), which is a common cause of disability and mortality, is an important threat to human health, and the annual global incidence of CA is (36-128)/100,000 individuals (2).

In China, the incidence of CA is $\sim 41.8 / 100,000$ and CA results in $\sim 544,000$ cases of mortality annually (3), which is the highest rate worldwide. In addition, at present, only $1 \%$ of patients with CA occurring outside a hospital environment survive (4). Therefore, $\mathrm{CA}$ is considered a major issue that requires attention worldwide.

With the increasing public knowledge of cardiopulmonary resuscitation (CPR), the continued improvement of rescue technology, and improvements to emergency network construction, the probability of a patient with CA occurring outside a hospital environment to recover due to restoration of spontaneous circulation (ROSC) is increasing (5). However, patients with CA who undergo CPR outside a hospital environment may suffer ischemic-hypoxic injury, particularly in the vulnerable regions of the hippocampus, cortex, thalamus and cerebellum. Ischemia may occur even if ROSC is achieved, and may result in necrosis and apoptosis of neurons, thereby leading to neurological damage and disorders including learning difficulties, anterograde amnesia, coma, a persistent vegetative state or mortality (6-9). The success or failure of brain recovery may be dependent on the quality of life of patients. In addition, numerous researchers advocate a change from CPR to cardiopulmonary cerebral resuscitation (CPCR). However, how to conduct CPCR so as to reduce or avoid neuronal damage and promote neurological recovery remains to be elucidated.

Mesenchymal stem cells (MSCs) are bone marrow-derived non-hematopoietic stem cells, which have been successfully applied in various types of tissue repair, first observed by Friedenstein et al in 1976 (10). MSCs have the potential for multi-directional differentiation, and can self-renew and differentiate into numerous types of cell depending on the microenvironment, including osteoblasts, chondroblasts, adipocytes and muscle cells $(11,12)$. 
Previous studies have focused on the application of MSCs for the repair of nerve tissue in models of focal cerebral ischemia; however, whole brain ischemic injury may occur following CA $(13,14)$. There have been few studies regarding MSC transplantation for the treatment of global ischemia, particularly as a result of $\mathrm{CA}$; however, they may reduce brain damage and improve neurological function (15-17). A previous study investigated MSC transplantation into the brain and demonstrated that MSCs were able to differentiate into neuron-like cells (18). The MSCs were administered to a rat model following CPR via vascular pathways, using magnetic resonance imaging in vivo tracking technology to observe the MSCs passing through the blood-brain barrier. MSCs have been shown to undergo directional migration to the hippocampus and temporal cortex, regions that are particularly vulnerable following global cerebral ischemia (19), and have been shown to reduce brain tissue damage to these areas, which may promote functional recovery (20).

In the present study, MSCs were labeled with 4'6-diamidino-2-phenyl-indole (DAPI) fluorescent dye and transplanted into rats following CPR using one of three methods: Stereotaxic infusion into the lateral ventricle (LV), infusion to the internal carotid artery (A), and infusion to the femoral vein (V). The migration and distribution of MSCs in the brain, and their effects on brain cell damage and neurological recovery were observed. Furthermore, the effects of various methods of MSC transplantation on cerebral resuscitation of the rats following CA were investigated, in order to screen the most effective migratory pathway.

\section{Materials and methods}

Experimental animals. A total of 40 Sprague-Dawley (SD) rats (age, 4-5 weeks; weight, 100-150 and 300-400 g) were obtained from the Laboratory Animal Center of Sun Yat-sen University (Guangdong, China). Their housing conditions consisted of a $12 \mathrm{~h}$ light/dark cycle, $40-70 \%$ humidity (20-24 $4^{\circ}$ ) and ad libitum access to food and water. The study was approved by the Animal Ethics Committee of Sun Yat-sen University.

Culture of MSCs. In the present study, the method of adherent cell culture was adopted. SD rats were anesthetized using $\mathrm{CO}_{2}$ inhalation and sodium pentobarbital $(45 \mathrm{~g} / 1 ; 0.1 \mathrm{ml} / 100 \mathrm{~g}$; Sigma-Aldrich, St. Louis, MO, USA) intraperitoneal injection prior to their sacrifice by cervical dislocation. Then, rats were placed into $75 \%$ ethanol for $\sim 5 \mathrm{~min}$ for disinfection. Subsequently, the rats were removed from the ethanol under sterile conditions, and in a laminar flow cabinet, lower limb muscles and soft tissue were cut, and the femur and tibia from each side were removed and placed in petri dishes.

The ends of the femur and tibia were cut and flushed with fetal calf serum-free Dulbecco's modified Eagle's medium (DMEM; Gibco; Thermo Fisher Scientific, Inc., Waltham, MA, USA)/Ham's F12, the bone marrow cavity was repeatedly washed until the entire bone marrow cavity was white.

The fluid used for flushing was placed into a centrifuge tube and centrifuged at $600 \mathrm{x} \mathrm{g}$ for $5 \mathrm{~min}$. The supernatant was removed and thoroughly mixed with DMEM $(8 \mathrm{ml}$ for each rat) following centrifugation. The mixture $(4 \mathrm{ml})$ was placed into $25-\mathrm{cm}^{2}$ flasks and stored in three incubators at $37^{\circ} \mathrm{C}$ in an atmosphere containing $5 \% \mathrm{CO}_{2}$ with saturated humidity. After $24 \mathrm{~h}$, half the media were changed, and after $48 \mathrm{~h}$, all of the media were changed, this was repeated every other day throughout the experiment. The media were aspirated when the cells had grown to $90 \%$ cell confluence ( $\sim 6-7$ days), and the flask was rinsed once with phosphate-buffered saline (PBS). Trypsin $(0.25 \%, 3 \mathrm{ml})$ containing $0.1 \mathrm{mmol} / 1$ EDTA was added to the culture flasks for $2 \mathrm{~min}$. Trypsin digestion was observed under an inverted microscope (T2000U; Nikon Corporation, Tokyo, Japan); when the MSCs were round and separated, DMEM was added to terminate the digestion.

The media were removed and the flask was washed, prior to centrifugation at $600 \mathrm{x}$ g for $5 \mathrm{~min}$. The supernatant was removed and the cell pellet was resuspended in DMEM (1:2, respectively). This process was repeated when the culture next reached $\sim 90 \%$ confluence ( $\sim 2-3$ days). Cells from passage 3 (P3) were digested with $0.25 \%$ trypsin and washed three times with PBS. Fluorescent-labeled antibodies against cluster of differentiation (CD)44 (cat. no. 561860), CD34 (cat. no. 551387), CD45 (cat. no. 561087) and CD90 (cat. no. 553012) (all diluted 1:250; all purchased from BD Pharmigen, San Diego, CA, USA) were added and incubated at $4^{\circ} \mathrm{C}$ for $30 \mathrm{~min}$. The unbound antibodies were removed by washing with PBS, and the cells were fixed with $1 \%$ paraformaldehyde (Sigma-Aldrich) for $15 \mathrm{~min}$. Surface antigen expression of the MSCs was detected by flow cytometry (BD FACSCalibur; BD Biosciences, Franklin Lakes, NJ, USA).

In addition, MSCs from P3 were stained with $4 \mathrm{ml}$ DMEM supplemented with nuclear stain solution containing $50 \mu \mathrm{g} / \mathrm{ml}$ DAPI (Biomol GmbH, Hamburg, Germany) for $2 \mathrm{~h}$ at $37^{\circ} \mathrm{C}$ in a humidified incubator containing $5 \% \mathrm{CO}_{2}$. After staining with DAPI, the MSCs were observed under a fluorescence microscope (Nikon Eclipse TiU; Nikon Corporation). These cells were then prepared for transplantation.

Grouping. Prior to the establishment of a CA resuscitation model, rats were randomly divided into four groups, which were subdivided into 3 and 7 day subgroups. Asphyxiation was used to generate a CA resuscitation model. The groups $(n=8)$ were as follows: i) Control group, rats received CPR without MSC transplantation; ii) LV group, MSC transplantation was performed into the lateral ventricle with a stereotaxic instrument following ROSC; iii) A group, MSC transplantation via the inner side of the carotid artery following ROSC; and iv) V group, MSC transplantation was performed via the side of the femoral vein following ROSC. In each transplantation group, MSC transplantation was conducted with $\sim 1 \times 10^{6}$ cells 1 h after ROSC.

Transplantation process. Healthy male SD rats (weight, 300-400 g) underwent fasting for $24 \mathrm{~h}$ with ad libitum access to water prior to development of the model. Prior to surgery, anesthesia was induced by $\mathrm{CO}_{2}$ inhalation and sodium pentobarbital $(45 \mathrm{~g} / \mathrm{l} ; 0.1 \mathrm{ml} / 100 \mathrm{~g})$ intraperitoneal injection. Bilateral skin preparation was performed on the chest, back and groin of the rats, which were fixed on the operating table in the supine position. Tracheal intubation was conducted using a medical ear, nose and throat mirror and a focus light under direct vision. The tracheal tube was fixed to the lower lip area 
following successful tracheal intubation and attached to a $\mathrm{CO}_{2}$ concentration monitor (Capstar-100; CWE, Inc., Ardmore, PA, USA), in order to continuously monitor end-expiratory carbon dioxide $\left(\mathrm{ETCO}_{2}\right)$ levels. Following disinfection, the lower limb was cut and the femoral artery was dissected and ligated with a surgical suture, subsequent to blocking the ophthalmic artery blood flow with tweezers. A cut was made above the femoral artery ligation point with ophthalmic scissors, and a heparinzation polyethylene pipe (Beckton Dickinson UK, Ltd., Oxford, UK) was inserted into the cut and into the thoracic aorta along the femoral artery $(3-4 \mathrm{~cm})$. Subsequently, the ductus arteriosus was fixed with a suture and the arterial catheter was connected to a multichannel analyzer (Spacelabs Healthcare, Snoqualmie, WA, USA) containing pressure sensors for physiological parameters, for the continuous monitoring of mean arterial pressure (MAP). A temperature sensor was inserted into the anus of the rats at a depth of 3-4 cm for continuous monitoring of temperature changes and a metal needle was inserted subcutaneously in the upper limbs and the left lower limb and connected to an electrocardiography (ECG) machine (Spacelabs Healthcare) for continuous ECG monitoring.

Therapeutic agent-induced asphyxiation and $C A$ resuscitation procedures. Following surgery, the rats were allowed to fully awaken and baseline physiological parameters were recorded. A respiratory inhibitor, vecuronium (Zhejiang Xianju Pharmaceutical Co., Ltd., Taizhou, China), was injected at a concentration of $1.0 \mathrm{mg} / \mathrm{kg}$ in the designated door of a T-branch pipe connected to the arterial catheter. A total of $10 \mathrm{sec}$ after injection, the rats gradually stopped breathing. Subsequently, changes in blood pressure were observed in the rats; reduced MAP to $20 \mathrm{mmHg}$ in 4-5 min is considered a criterion for CA. The ventilator was opened and adjusted, and the device parameters for chest compression were set to ensure the pressing head was adjusted to the lower sternum of the rat and the lower end was located on the top $2.5 \mathrm{~cm}$ of the xiphoid. Following $5 \mathrm{~min}$ and $45 \mathrm{sec}$ of CA, the endotracheal tube was connected to the ventilator with oxygen ventilation, a tidal volume of $0.6 \mathrm{ml} / 100 \mathrm{~g}$ and a respiratory rate of 100 beats/min. Following 6 min of CA, the chest compressions were conducted using a CPR device (developed independently by the Institute of Cardiopulmonary Cerebral Resuscitation, Sun Yat-sen University) with a pressing frequency of 200 times/min, a ratio of pressing to ventilation of $2: 1$ and a compression depth of $1 / 3$ of the anteroposterior diameter. Following $2 \mathrm{~min}$ of compression, $0.1 \mathrm{ml}$ adrenaline was injected via the femoral artery with $0.1 \mathrm{ml}$ saline and heparin. Continuous chest compressions and ventilation were conducted until the MAP was maintained at $>60 \mathrm{mmHg}$ for $5 \mathrm{~min}$, which is a criteria for ROSC. Pure oxygen aeration was conducted for $15 \mathrm{~min}$, followed by mechanical ventilation with $50 \%$ oxygen. If, following $5 \mathrm{~min}$ of chest compressions, the rats failed to achieve ROSC, the recovery was considered a failure and the rat was excluded from the study. Following ROSC, the rats received continuous mechanical ventilation for a further $30 \mathrm{~min}$ and hemodynamic changes were continuously monitored.

Transplantation of MSCs following recovery. DAPI-labeled MSCs were administered to the rats $1 \mathrm{~h}$ after ROSC according to their groups via the lateral cerebral ventricles using a stereotaxic instrument, via the internal carotid artery, or by femoral vein injection. Subsequently, all catheters were removed and $250 \mathrm{~g} / \mathrm{l}$ (0.2 ml) cephalosporin VI (Beijing Tsinghua Unisplendour Pharmaceutical Factory, Beijing, China) was administered by intramuscular injection, and the rats were returned to the animal cages. On the first day following ROSC, rats were housed indoors individually, maintained at room temperature $\left(\sim 25^{\circ} \mathrm{C}\right)$ and artificially fed every $6 \mathrm{~h}$ with $5 \%$ dextrose saline injection. After 3 days the rats were allowed to return to the group cage.

Detailed records of survival following ROSC were kept. At the end of the experiment, the rat mortality and time, and survival rates were statistically analyzed.

Assessment of neurological function in rats. The experimental rats were scored on days 1, 3 and 7 following ROSC using the Neurological Deficit Scores (NDS) as a standard (21). NDS were used to grade brain functions, such as awakening, cranial nerve reflex, motor function and the simple act of reflection. Scores range from 0 to 80 points; a score of 0 represents brain death and a score of 80 represents normal brain function.

Pathological examination of the hippocampus and temporal cortex. Normal, healthy, nerve cells were cut using a microtome (Leica RM2235; Leica Biosystems, Wetzlar, Germany) and sections $(4 \mu \mathrm{M})$ were stained with hematoxylin and eosin (H\&E; Wuhan Boster Biotechnology, Ltd., Wuhan, China) and observed under a microscope (T2000U; Nikon, Tokyo, Japan).

Blood specimen collection. Anesthesia was induced with $\mathrm{CO}_{2}$ and the rats were then sacrificed using an intraperitoneal injected of sodium pentobarbital $(45 \mathrm{~g} / 1 ; 0.1 \mathrm{ml} / 100 \mathrm{~g})$. The rats were fixed on an operating table, the abdominal wall was opened and the inferior vena cava was separated. A syringe was used to take $5 \mathrm{ml}$ blood samples from the inferior vena cava, the blood samples were then injected into a drying tube for $10 \mathrm{~min}$, and centrifuged at 1,200 $\mathrm{x} g$ for $15 \mathrm{~min}$. The supernatant was placed into a centrifuge tube and maintained at $-80^{\circ} \mathrm{C}$ to preserve the S100 calcium-binding protein B (S100B) levels in the serum, which were uniformly analyzed at the end of the experiment.

Detection of brain water content. After blood specimens were collected, the chest was exposed and paraformaldehyde was injected from the internal carotid artery to the brain, until the superior vena cava returned clear paraformaldehyde. Following paraformaldehyde perfusion, the rats were cervically dislocated, the skull was opened and the right side of the brain was removed. The right side of the brain was removed and the wet-weight was determined using an analytical balance. The tissue was then dried in an oven at $105^{\circ} \mathrm{C}$ for $48 \mathrm{~h}$ and the dry weight was measured. The water content of the brain was calculated according to the Elliot formula: Wet weight - dry weight)/wet weight $\mathrm{x} 100 \%$. Determination of S100B protein in the sera was conducted using an enzyme-linked immunosorbent assay (ELISA; Wuhan Huamei Biotech Co., Ltd., Wuhan, China).

Statistical analysis. Statistical analyses were conducted using SPSS version 20.0 for Mac (IBM SPSS, Armonk, NY, USA) and the data are presented as the mean \pm standard deviation. Groups were compared using analysis of variance and the $\chi^{2}$ test. $\mathrm{P}<0.05$ was considered to indicate a statistically significant difference. 

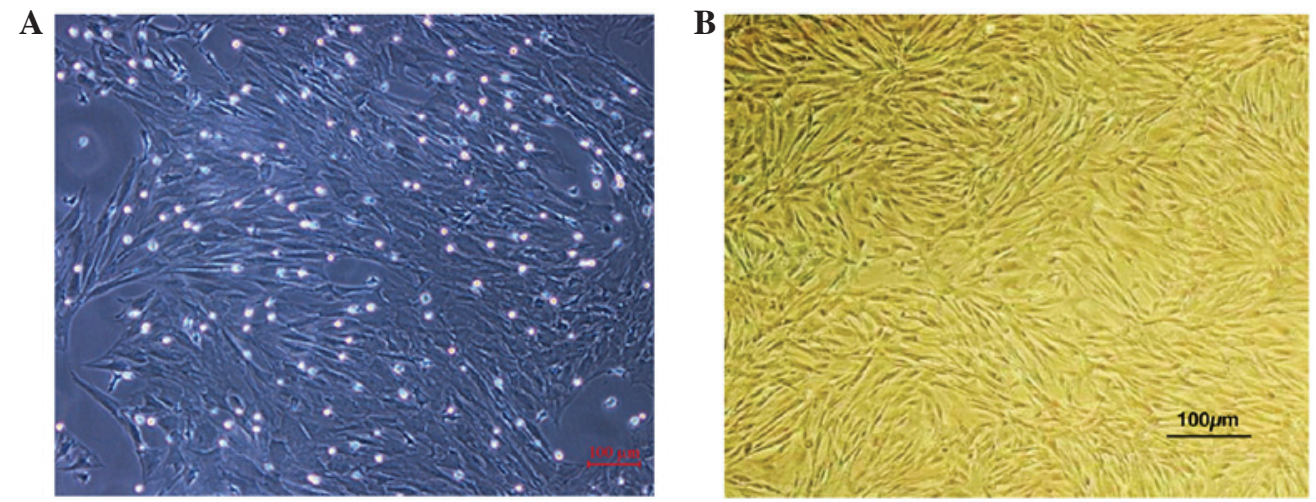

Figure 1. Cell morphology of cultured primary and P3 generation mesenchymal stem cells. (A) Primary cell culture at 7 days; large, single, spindle cell colonies were observed with greater cell impurities. (B) P3 cells were evenly distributed with fewer cell impurities. Magnification, x100. P3, passage 3.

A

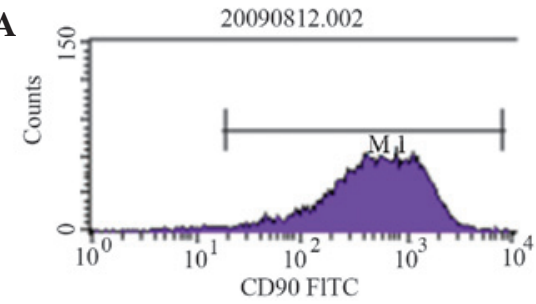

File:20090812.002

Gate:G1

\begin{tabular}{lll} 
Marker $\%$ Gated & Mean \\
\hline Al & 100.00 & 658.58
\end{tabular}

M1 $\quad 99.00 \quad 663.76$

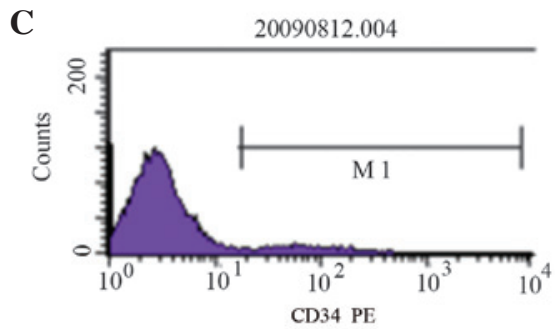

File:20090812.004

Gate:G1

\begin{tabular}{lll} 
Marker & $\%$ Gated & Mean \\
\hline Al & 100.00 & 7.45 \\
M1 & 5.10 & 86.08
\end{tabular}

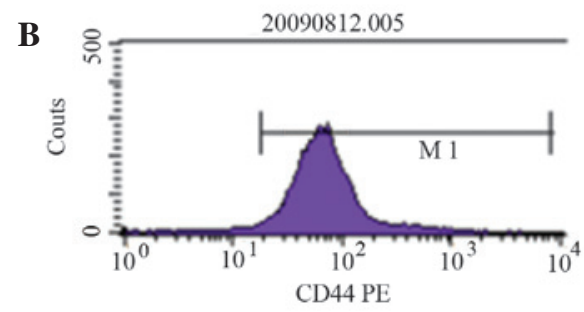

File:20090812.005

Gate:G1

\begin{tabular}{lcc} 
Marker & $\%$ Gated & Mean \\
\hline Al & 100.00 & 83.22 \\
M1 & 97.60 & 84.97
\end{tabular}

D

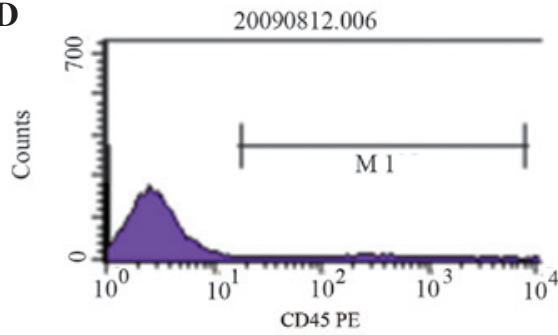

File:20090812.006

Gate:Gl

\begin{tabular}{lcc} 
Marker & \% Gated & Mean \\
\hline A1 & 100.00 & 28.40 \\
M1 & 4.96 & 407.74
\end{tabular}

Figure 2. Flow cytometric analysis of cell surface markers on passage 3 mesenchymal stem cells. (A) CD90 expression rate was $99.00 \%$, (B) CD44 expression rate was $97.60 \%$, (C) CD34 expression rate was 5.10\% and (D) CD45 expression rate was 4.96\%. CD, cluster of differentiation; FITC, fluorescein isothiocyanate; PE, phycoerythrin.

\section{Results}

Isolation, cultivation and identification of MSCs. The P3 MSCs demonstrated uniform cell morphology as presented in Fig. 1A and B. Stromal cell surface markers were detected by flow cytometry; CD90 expression was detected in $99.00 \%$ of cells and CD44 expression was detected in $97.60 \%$ of cells (Fig. 2A and B). Hematopoietic cell surface markers were also observed; CD34 expression was detected in $5.10 \%$ of cells and CD45 expression was detected in $4.96 \%$ of cells (Fig. 2C and D). These results indicate that MSCs obtained in the present study were of a high purity following isolation, culture and passage.
Observation of MSCs fluorescently labeled with DAPI. The transplanted MSCs were observed under a fluorescence microscope, the results demonstrated that the rate of DAPI staining was $99 \%$, suggesting that DAPI may be used as a tracer to label MSCs for in vivo transplantation (Fig. 3).

Monitoring of recovering rats. Physiological parameters were monitored in each group prior to and 5, 10, 20 and $30 \mathrm{~min}$ following ROSC. As presented in Tables I and II, there were no significant differences between the MAP, heart rate and $\mathrm{ETCO}_{2}$ of rats in each group at the end of the surgical procedure or after ROSC. 
Table I. Comparison of baseline MAP, $\mathrm{HR}$ and $\mathrm{ETCO}_{2}$ levels in each group (mean \pm standard deviation).

\begin{tabular}{lccr}
\hline Group & MAP $(\mathrm{mmHg})$ & HR $(\mathrm{bpm})$ & $\mathrm{ETCO}_{2}(\mathrm{mmHg})$ \\
\hline Control $(\mathrm{n}=8)$ & $133.63 \pm 11.19$ & $383.50 \pm 15.03$ & $35.34 \pm 4.32$ \\
V $(\mathrm{n}=8)$ & $129.13 \pm 8.43$ & $389.25 \pm 19.61$ & $39.01 \pm 6.70$ \\
A $(\mathrm{n}=8)$ & $133.25 \pm 9.49$ & $400.38 \pm 16.55$ & $38.85 \pm 5.68$ \\
LV $(\mathrm{n}=8)$ & $122.75 \pm 9.94$ & $388.87 \pm 25.40$ & $37.51 \pm 6.42$ \\
F-value & 2.121 & 1.051 & 0.674 \\
P-value & 0.120 & 0.386 & 0.575
\end{tabular}

MAP, mean arterial pressure; $\mathrm{HR}$, heart rate; $\mathrm{ETCO}_{2}$, end-expiratory $\mathrm{CO}_{2} ; \mathrm{V}$, femoral venous infusion; A, intra-carotid administration; LV, lateral cerebral ventricle administration.

Table II. Comparison of MAP, $\mathrm{HR}$ and $\mathrm{ETCO}_{2}$ levels in each group at various time-points following ROSC (mean \pm standard deviation).

\begin{tabular}{|c|c|c|c|c|c|c|c|}
\hline Parameter & Time (ROSC) & $\begin{array}{l}\text { Group Con } \\
\quad(n=8)\end{array}$ & $\begin{array}{c}\text { Group V } \\
(n=8)\end{array}$ & $\begin{array}{c}\text { Group A } \\
(n=8)\end{array}$ & $\begin{array}{c}\text { Group LV } \\
(\mathrm{n}=8)\end{array}$ & F-value & P-value \\
\hline \multirow[t]{4}{*}{ MAP (mmHg) } & $5 \mathrm{~min}$ & $113.63 \pm 19.09$ & $119.13 \pm 26.64$ & $136.38 \pm 15.73$ & $127.00 \pm 12.03$ & 2.136 & 0.118 \\
\hline & $10 \mathrm{~min}$ & $134.13 \pm 21.86$ & $122.20 \pm 15.12$ & $140.45 \pm 13.52$ & $135.33 \pm 20.32$ & 1.465 & 0.246 \\
\hline & $20 \mathrm{~min}$ & $107.58 \pm 23.09$ & $101.08 \pm 15.56$ & $111.92 \pm 14.27$ & $110.96 \pm 13.18$ & 0.667 & 0.580 \\
\hline & $30 \mathrm{~min}$ & $103.75 \pm 10.36$ & $98.63 \pm 9.04$ & $102.50 \pm 7.39$ & $101.81 \pm 10.39$ & 0.340 & 0.797 \\
\hline \multirow[t]{4}{*}{ HR (bpm) } & $5 \mathrm{~min}$ & $308.13 \pm 25.72$ & $295.38 \pm 19.84$ & $312.13 \pm 31.48$ & $307.50 \pm 23.35$ & 0.646 & 0.592 \\
\hline & $10 \mathrm{~min}$ & $322.75 \pm 44.44$ & $313.75 \pm 30.70$ & $316.75 \pm 28.47$ & $310.38 \pm 26.95$ & 0.205 & 0.892 \\
\hline & $20 \mathrm{~min}$ & $285.89 \pm 36.42$ & $285.25 \pm 44.56$ & $306.00 \pm 14.98$ & $308.38 \pm 27.69$ & 1.167 & 0.340 \\
\hline & $30 \mathrm{~min}$ & $289.00 \pm 35.96$ & $293.50 \pm 31.38$ & $303.63 \pm 11.09$ & $304.63 \pm 20.82$ & 0.304 & 0.822 \\
\hline \multirow[t]{4}{*}{$\mathrm{ETCO}_{2}(\mathrm{mmHg})$} & $5 \mathrm{~min}$ & $50.98 \pm 12.40$ & $53.11 \pm 6.70$ & $60.46 \pm 7.33$ & $56.49 \pm 10.19$ & 1.542 & 0.225 \\
\hline & $10 \min$ & $50.61 \pm 8.42$ & $52.51 \pm 8.42$ & $57.78 \pm 8.45$ & $55.94 \pm 7.97$ & 1.215 & 0.323 \\
\hline & $20 \mathrm{~min}$ & $56.99 \pm 5.25$ & $59.11 \pm 7.32$ & $60.15 \pm 8.94$ & $51.83 \pm 8.67$ & 1.854 & 0.160 \\
\hline & $30 \mathrm{~min}$ & $49.38 \pm 5.59$ & $52.38 \pm 5.57$ & $57.78 \pm 6.13$ & $53.00 \pm 7.65$ & 2.441 & 0.085 \\
\hline
\end{tabular}

ROSC, restoration of spontaneous circulation; MAP, mean arterial pressure; HR, heart rate; $\mathrm{ETCO}_{2}$, end-expiratory $\mathrm{CO}_{2}$; Con, control; V, femoral venous infusion; A, intra-carotid administration; LV, lateral cerebral ventricle administration.

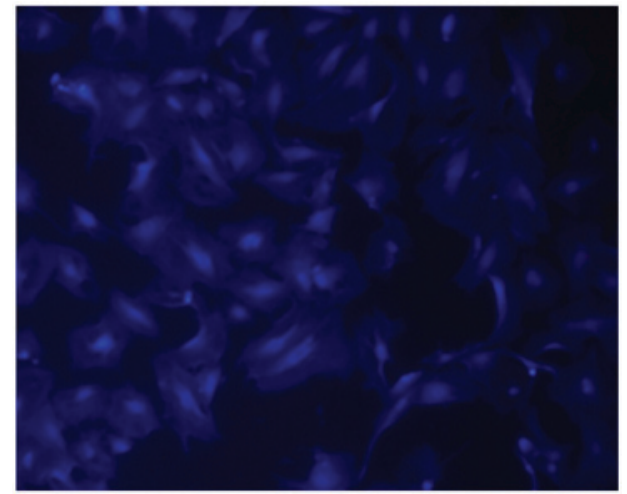

Figure 3. Passage 3 mesenchymal stem cells labeled with 4'6-diamidino-2-phenyl-indole (magnification, x200).

Distribution and migration of transplanted MSCs. Following transplantation of MSCs, the blue fluorescent-labeled cells were observed in the brain on day 3 post-ROSC. The MSCs were predominantly distributed in the hippocampus and temporal cortex, which is consistent with global cerebral ischemia. On day 3 following ROSC, the number of MSCs in the LV group was significantly greater, as compared with the $\mathrm{A}$ and $\mathrm{V}$ groups $(\mathrm{P}<0.01)$. There was no significant difference between the $\mathrm{A}$ and $\mathrm{V}$ groups $(\mathrm{P}>0.05)$. On day 7 following ROSC, a greater number of MSCs in the LV group were observed in the hippocampal region, as compared with in the $\mathrm{A}$ and $\mathrm{V}$ groups $(\mathrm{P}<0.05)$; however, there was no difference in the number of MSCs in the temporal cortex regions between the various transplant groups (P>0.05; Figs. 4 and 5).

Measurement of physiological parameters in experimental rats. Mortality rate. One rat in the control group survived only 1 day following ROSC; however, there were no mortalities in the experimental groups.

Weight. The weight of the rats declined in each group following ROSC; however, differences in weight between days 3 and 7, 

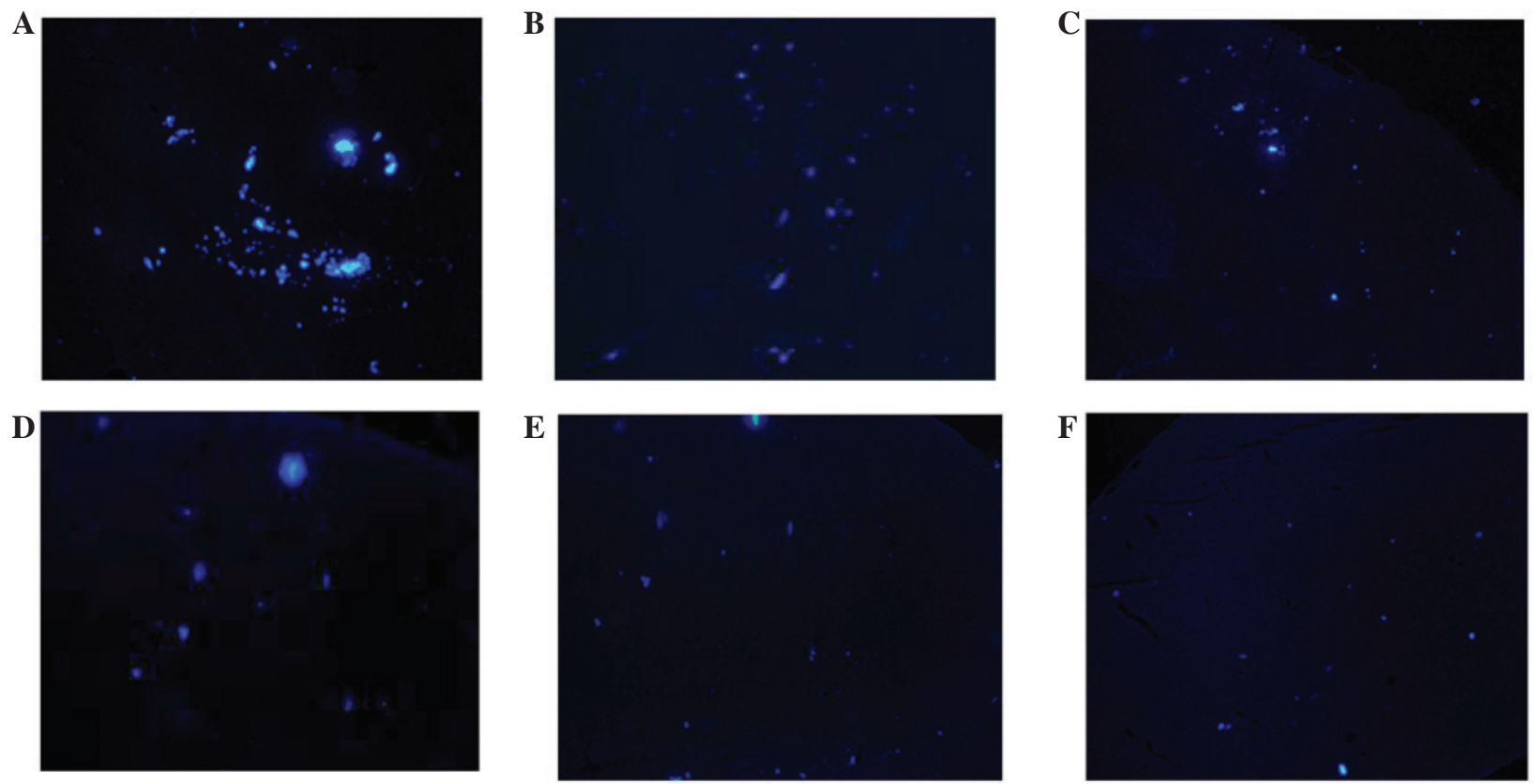

Figure 4. Visible blue fluorescent-labeled MSCs in the hippocampus and temporal cortex 3 days after MSC transplantation. (A) Hippocampus and (B) temporal cortex of the lateral cerebral ventricle administration group; (C) hippocampus and (D) temporal cortex of the intra-carotid administration group; and (E) hippocampus and (F) temporal cortex of the femoral venous infusion group. Magnification, x100. MSCs, mesenchymal stem cells.

A

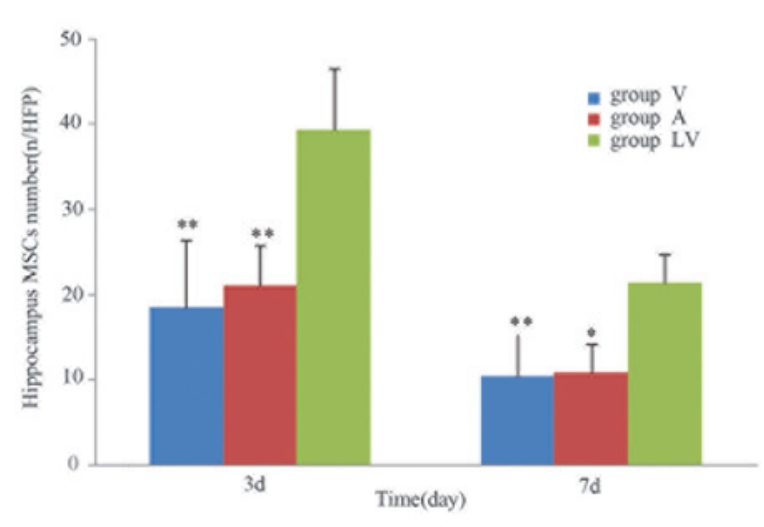

B

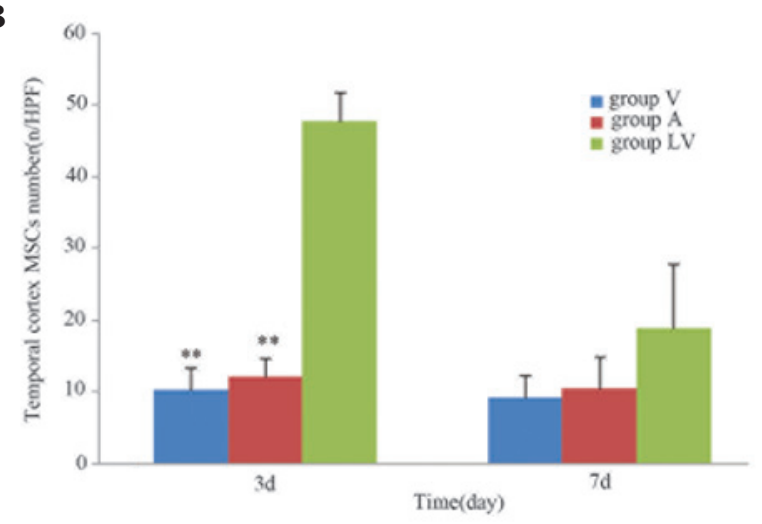

Figure 5. Distribution of cells from each MSC transplantation group in the (A) hippocampus and (B) temporal cortex. Data are presented as the mean \pm standard deviation."P $<0.05$ vs. the LV group; ${ }^{* *} \mathrm{P}<0.01$ vs. the $\mathrm{LV}$ group. MSC, mesenchymal stem cell; V, femoral venous infusion; A, intra-carotid administration; $\mathrm{LV}$, lateral ventricle administration, HPF, high power field.

and prior to the experiment were not statistically significant (P>0.05; Table III).

NDSS. Neurological scores of the rats were not statistically different among the groups 1 day after ROSC $(\mathrm{P}>0.05)$. However, 3 and 7 days after ROSC, the NDSS were significantly higher in the MSC transplantation groups, as compared with those in the control group $(\mathrm{P}<0.05)$, and were significantly higher in the LV group than in the $\mathrm{A}$ and $\mathrm{V}$ groups $(\mathrm{P}<0.05)$. There was no significant difference between the $\mathrm{A}$ and V groups ( $\mathrm{P}>0.05$; Fig. 6).

\section{Pathological examination of the hippocampus and temporal} cortex with HE staining. Following staining of the normal, healthy, nerve cells with HE they were observed under a microscope; nuclei were stained lightly, no vacuoles were observed, and axons visibly extended to the surrounding cells. However,

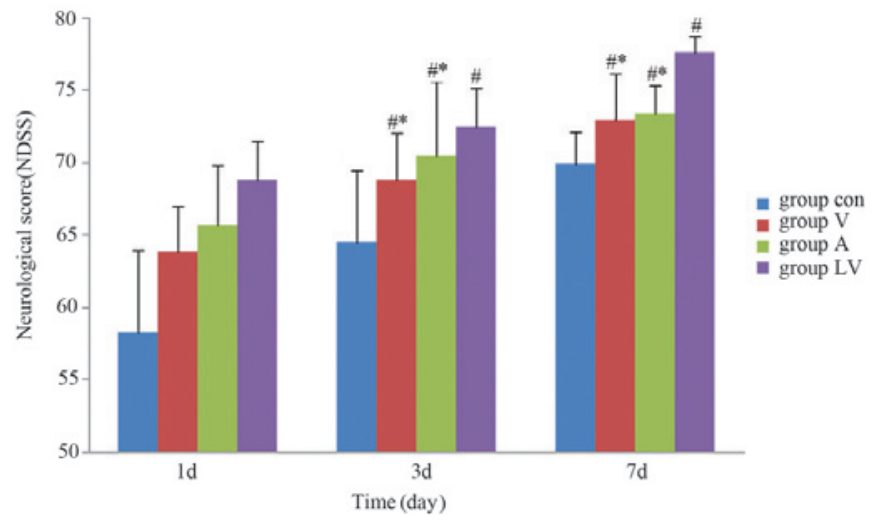

Figure 6. Neurological score of the rats in each group following return of spontaneous circulation. Data are presented as the mean \pm standard deviation. ${ }^{~} \mathrm{P}<0.05$ vs. the Con group; ${ }^{\mathrm{P}} \mathrm{P}<0.05$ vs. the $\mathrm{LV}$ group. Con, control; V, femoral venous infusion; A, intra-carotid administration; LV, lateral ventricle administration; NDSS, Neurological Deficit Scale Scores. 
Table III. Comparison of weight following recovery at various time-points among the groups (mean \pm standard deviation).

\begin{tabular}{lcccccr}
\hline Time & Group Con & Group V & Group A & Group LV & F-value & P-value \\
\hline Prior to recovery & $364.87 \pm 19.67$ & $348.78 \pm 20.65$ & $348.50 \pm 23.88$ & $351.87 \pm 22.99$ & 0.87 & 0.47 \\
ROSC 3 days & $321.37 \pm 21.66$ & $308.65 \pm 19.64$ & $310.78 \pm 26.41$ & $308.13 \pm 27.00$ & 0.47 & 0.70 \\
ROSC 7 days & $277.00 \pm 10.12$ & $273.75 \pm 11.78$ & $287.00 \pm 10.93$ & $285.75 \pm 19.07$ & 0.72 & 0.57 \\
\hline
\end{tabular}

Con, control; V, femoral venous infusion; A, intra-carotid administration; LV, lateral cerebral ventricle administration; ROSC, restoration of spontaneous circulation.

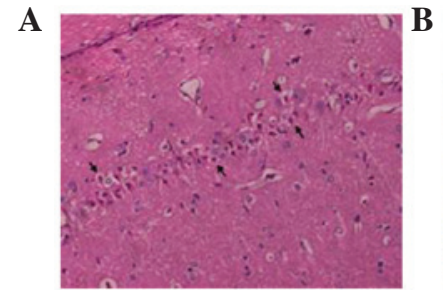

$\mathbf{E}$

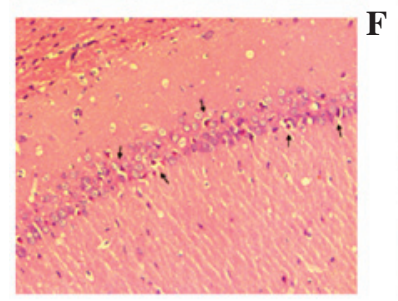

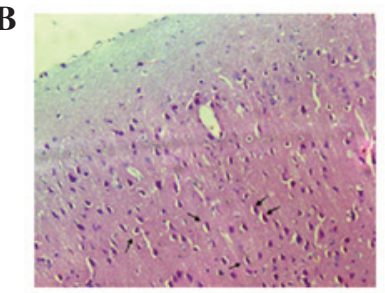

F

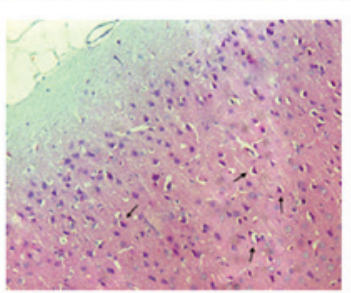

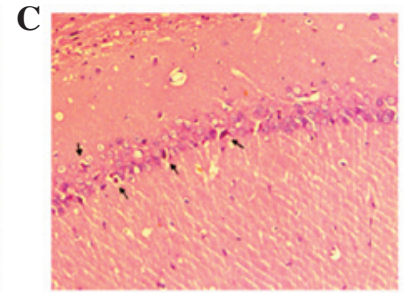
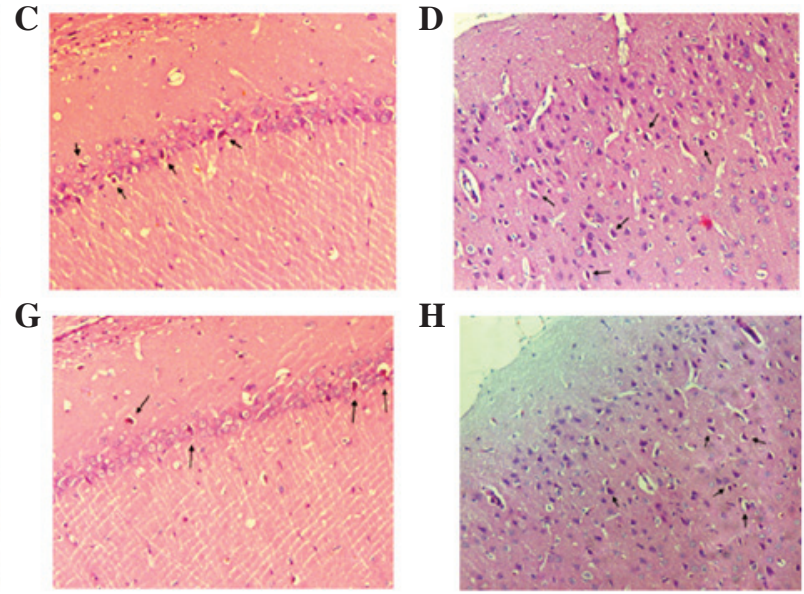

Figure 7. Pathological examination of hematoxylin and eosin staining in the hippocampal CA1 and the temporal cortex 7 days after return of spontaneous circulation (magnification, x200). In each recovery group there was strong nuclear staining, vacuolar changes associated with nerve damage, and eosinophilic cells (indicated with arrows). (A) CA1 and (B) temporal cortex in the control group, (C) CA1 and (D) temporal cortex in the intra-carotid administration group, (E) CA1 and (F) temporal cortex in the femoral venous infusion group, and (G) CA1 and (H) temporal cortex in the lateral ventricle administration group. CA1, region I of hippocampus proper.

rats with global cerebral ischemia following CPR exhibited nerve cell damage visible in regions of the hippocampus and the temporal cortex. The damage was demonstrated predominantly by nuclear condensation, and vacuolar alterations around the nucleus, which was were called eosinophilic-like changes (Fig. 7). The number of cells exhibiting these changes were significantly lower in the hippocampus and temporal cortex of the MSC transplantation groups, as compared with in the control group $(\mathrm{P}<0.01)$. In addition, nerve damage was significantly reduced in the LV group, as compared with in the $\mathrm{A}$ and $\mathrm{V}$ groups $(\mathrm{P}<0.01)$; however, there was no significant difference between the A and V groups ( $\mathrm{P}>0.05$; Fig. 8).

Detection of brain water content. The brain water content was significantly lower in the MSC groups than in the control group 3 and 7 days after ROSC $(\mathrm{P}<0.01)$. The content was significantly lower in the LV group than in group A $(\mathrm{P}<0.05)$ and group $\mathrm{V}(\mathrm{P}<0.01)$ on day 3 following ROSC. In addition, brain water content was significantly lower in the LV group, as compared with in the $\mathrm{A}$ and $\mathrm{V}$ groups $(\mathrm{P}<0.05) 7$ days following ROSC; however, there were no significant differences between groups $\mathrm{A}$ and $\mathrm{V}$ at either time ( $\mathrm{P}>0.05$; Fig. 9).

Detection of serum S100B levels by ELISA. Serum S100B levels were significantly increased in each group after ROSC. Serum S100B levels were significantly lower in each of the
MSC transplantation groups, as compared with in the control group 3 and 7 days after ROSC $(\mathrm{P}<0.05)$, serum S100B levels were significantly lower in the LV group than in groups $\mathrm{A}$ and V 3 days after ROSC $(\mathrm{P}<0.05)$; however, there was no significant difference between groups $\mathrm{A}$ and $\mathrm{V}(\mathrm{P}>0.05)$. S100B levels had decreased in each group 7 days after ROSC; however, there were no significant differences amongst the LV, $\mathrm{A}$ and $\mathrm{V}$ groups ( $\mathrm{P}>0.05$; Fig. 10).

\section{Discussion}

Global cerebral ischemia is a serious complication following CA, which directly affects patient prognosis following CPR. MSC transplantation is a novel therapeutic strategy used to treat ischemic injury, which has been used in the treatment of cerebral infarction and other nervous system diseases to therapeutic benefit (11). At present, very few studies have focused on MSC transplantation therapy for CA-induced global cerebral ischemia; however, previous studies have indicated that MSC transplantation may reduce brain damage and improve neurological function $(12,22,23)$. In the present study, DAPI-positive MSCs were predominantly observed in the regions of serious ischemic injury and in areas surrounding the hippocampus and temporal cortex 3 and 7 days after transplantation. In each MSC transplantation group, other parts of the brain exhibited no staining, indicating that the MSCs 

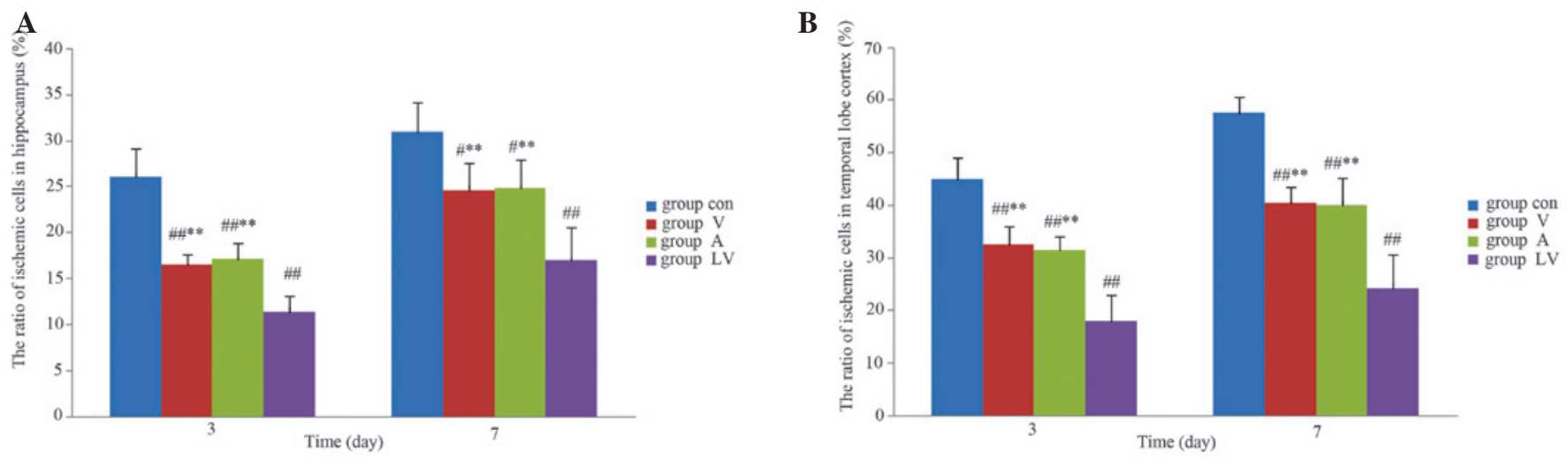

Figure 8. Nerve damage in the (A) hippocampus and (B) temporal cortex of rats in each resuscitation group. Data are presented as the mean \pm standard deviation. ${ }^{\#} \mathrm{P}<0.05$ vs. the Con group; ${ }^{\# /} \mathrm{P}<0.01$ vs. the Con group; ${ }^{* *} \mathrm{P}<0.01$ vs. the $\mathrm{LV}$ group. Con, control; V, femoral venous infusion; A, intra-carotid administration; LV, lateral ventricle administration.

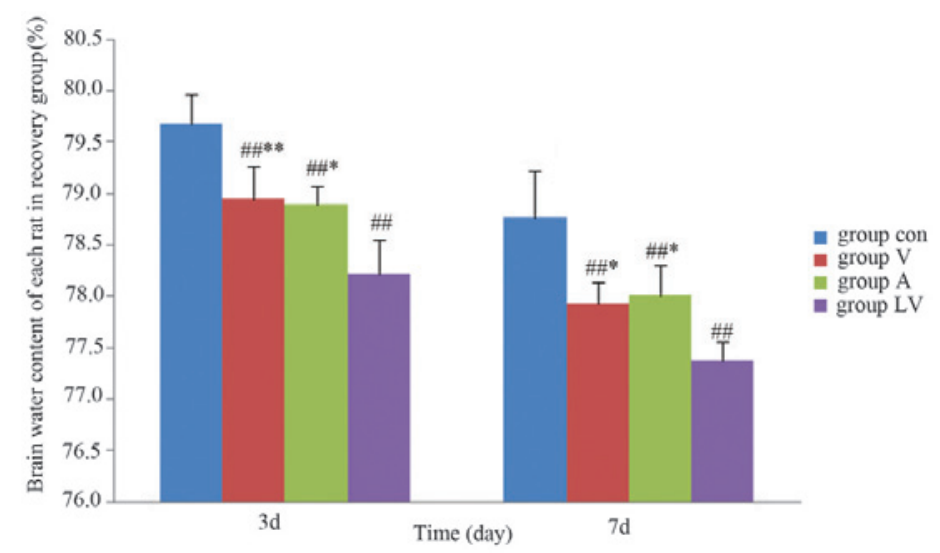

Figure 9. Brain water content of each transplantation group. Data are presented as the mean \pm standard deviation. ${ }^{\#} \mathrm{P}<0.05$ vs. the Con group; ${ }^{\# \#} \mathrm{P}<0.01$ vs. the Con group; ${ }^{*} \mathrm{P}<0.01$ vs. the LV group; ${ }^{* *} \mathrm{P}<0.05$ vs. the $\mathrm{LV}$ group. Con, control; $\mathrm{V}$, femoral venous infusion; $\mathrm{A}$, intra-carotid administration; $\mathrm{LV}$, lateral ventricle administration.

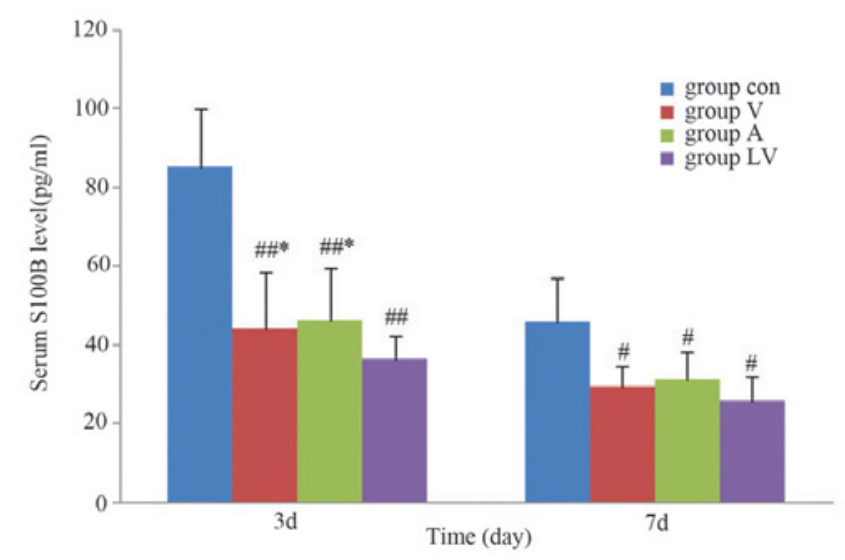

Figure 10. Detection of serum S100B levels in each recovery group. Data are presented as the mean \pm standard deviation. ${ }^{*} \mathrm{P}<0.05$ vs. the Con group; ${ }^{\# \#} \mathrm{P}<0.01$ vs. the Con group; "P $<0.05$ vs. the LV group. S100B, S100 calcium-binding protein B; Con, control; V, femoral venous infusion; A, intra-carotid administration; LV, lateral ventricle administration.

undergo regional distribution; these results are consistent with those of a previous in vitro study (24).

Pathological examination demonstrated that the number of damaged neurons in ischemic areas of the brain was significantly increased following CPR. Cell damage was predominantly pyknotic, and changes to the distribution of dye around the nucleus were detected after HE staining. The proportion of injured neurons in the hippocampus and temporal cortex was lower in the MSC transplantation groups, as compared with in the control group, thus suggesting that MSC transplantation may improve global ischemic injury and further promote recovery. The present study also 
demonstrated that brain water content was lower in the MSC transplantation groups than in the control group 3 and 7 days post-transplantation with MSCs, thus indicating that MSCs may also exert a therapeutic effect on brain edema.

The results of the present study demonstrated that the NDSS of rats in the MSC transplantation groups were higher than in the control group 3 and 7 days after ROSC, and the NDSS were close to normal levels by day 7 .

S100B protein, which is predominantly distributed in the glial and Schwann cells, is an injury-specific protein that appears following damage to the central nervous system (25). Hypoxic-ischemia injury to the brain may result in secondary damage to the blood-brain barrier, resulting in the release of S100B protein from the damaged nerve and glial cells into the blood circulation, thus causing increased serum levels. Measuring the levels of S100B following CA assesses the extent of brain damage, which is more sensitive for predicting the recovery of neurological function $(26,27)$. Results from the present study demonstrated that the protein levels of serum S100B in each group were significantly increased following ROSC; however, they were significantly decreased in the MSC transplantation groups, as compared with in the control group 3 and 7 days after ROSC.

In conclusion, MSCs may improve neurological recovery following injury via the repair of ischemic brain tissue. The present study indicated that MSC transplantation following CA and CPR had therapeutic benefits on global cerebral ischemia injury, and provides a basis for animal experiments regarding MSC transplantation for the recovery of brain function in patients with CA.

\section{Acknowledgements}

The present study was supported in part by a research grant from the Guangdong Provincial Department of Science and Technology of China (grant no. 2012B03180045).

\section{References}

1. Thom T, Haase N, Rosamond W, Howard VJ, Rumsfeld J, Manolio T, Zheng ZJ, Flegal K, O'Donnell C, Kittner S, et al: Heart disease and stroke statistics - 2006 update: A report from the American Heart Association Statistics Committee and Stroke Statistics Subcommittee. Circulation 113: e85-e151, 2006.

2. Grounds MD, White JD, Rosenthal N and Bogoyevitch MA: The role of stem cells in skeletal and cardiac muscle repair. J Histochem Cytochem 50: 589-610, 2002.

3. Grove JE, Lutzko C, Priller J, Henegariu O, Theise ND, Kohn DB and Krause DS: Marrow-derived cells as vehicles for delivery of gene therapy to pulmonary epithelium. Am J Respir Cell Mol Biol 27: 645-651, 2002.

4. Zi-Tong Huang. Improve the level of cardiopulmonary resuscitation measures and countermeasures. Zhonghuajizhenyixuezazhi 3: 153-154, 2004.

5. Bindslev L, Haack-Sørensen M, Bisgaard K, Kragh L, Mortensen S, Hesse B, Kjaer A and Kastrup J: Labelling of human mesenchymal stem cells with indium-111 for SPECT imaging: Effect on cell proliferation and differentiation. Eur J Nucl Med Mol Imaging 33: 1171-1177, 2006.

6. Wen $\mathrm{M}$ and Li SL: The studies of magnetic resonance imaging for molecular imaging. Zhongguo Yixue Yingxiang Jishu 1: 147-150, 2007 (In Chinese).

7. Jing XH, Yang L, Duan XJ, Xie B, Chen W, Li Z and Tan HB: In vivo MR imaging tracking of magnetic iron oxide nanoparticle labeled, engineered, autologous bone marrow mesenchymal stem cells following intra-articular injection. Joint Bone Spine 75: 432-438, 2008.
8. Fang X, Tang W, Sun S, Huang L, Huang Z and Weil MH: Mechanism by which activation of delta-opioid receptor reduces the severity of postresuscitation myocardial dysfunction. Crit Care Med 34: 2607-2612, 2006.

9. Hickey RW, Kochanek PM, Ferimer H, Alexander HL, Garman RH and Graham SH: Induced hyperthermia exacerbates neurologic neuronal histologic damage after asphyxial cardiac arrest in rats. Crit Care Med 31: 531-535, 2003.

10. Friedenstein AJ, Chailakhyan RK and Gerasimov UV: Bone marrow osteogenic stem cells: In vitro cultivation and transplantation in diffusion chambers. Cell Tissue Kinet 20: 263-272, 1987.

11. Joshi D and Behari M: Neuronal stem cells. Neurol India 51: 323-328, 2003.

12. Zheng W, Honmou O, Miyata K, Harada K, Suzuki J, Liu H, Houkin K, Hamada $\mathrm{H}$ and Kocsis JD: Therapeutic benefits of human mesenchymal stem cells derived from bone marrow after global cerebral ischemia. Brain Res 1310: 8-16, 2010.

13. Chen J, Li Y, Katakowski M, Chen X, Wang L, Lu D, Lu M, Cautam SC and Chopp M: Intravenous bone marrow stromal cell therapy reduces apoptosis and promotes endogenous cell proliferation after stroke in female rat. J Neurosci Res 73: 778-786, 2003.

14. Li Y, Chopp M, Chen J, Wang L, Gautam SC, Xu YX and Zhang Z: Intrastriatal transplantation of bone marrow nonhematopoietic cells improves functional recovery after stroke in adult mice. J Cereb Blood Flow Metab 20: 1311-1319, 2000.

15. Li N, Sarojini H, An J and Wang E: Prosaposin in the secretome of marrow stroma-derived neural progenitor cells protects neural cells from apoptotic death. J Neurochem 112: 1527-1538, 2010.

16. Li Y, Chopp M, Chen J, Wang L, Gautam SC, Xu YX and Zhang $\mathrm{Z}$ : Intrastriatal transplantation of bone marrow nonhematopoietic cells improves functional recovery after stroke in adult mice. J Cereb Blood Flow Metab 20: 1311-1319, 2000.

17. Tang WC and Sun SJ: Cardiopulmonary resuscitation and emergency cardiovascular Beijing: Beijing Science and Technology Press 572-586, 2008.

18. Barbash IM, Chouraqui P, Baron J, Feinberg MS, Etzion S, Tessone A, Miller L, Guetta E, Zipori D, Kedes LH, et al: Systemic delivery of bone marrow-derived mesenchymal stem cells to the infarcted myocardium: Feasibility, cell migration and body distribution. Circulation 108: 863-868, 2003.

19. Zhang W, Smith C, Howlett C and Stanimirovic D: Inflammatory activation of human brain endothelial cells by hypoxic astrocytes in vitro is mediated by IL-1beta. J Cereb Blood Flow Metab 20: 967-978, 2000.

20. Schoch HJ, Fischer S and Marti HH: Hypoxia-induced vascular endothelial growth factor expression causes vascular leakage in the brain. Brain 125: 2549-2557, 2002.

21. Geocadin RG, Ghodadra R, Kimura T, Lei H, Sherman DL, Hanley DF and Thakor NV: A novel quantitative EEG injury measure of global cerebral isehemia. Clin Neurophysiol 111: 1779-1187, 2000.

22. Perasso L, Cogo CE, Giunti D, Gandolfo C, Ruggeri P, Uccelli A and Balestrino $\mathrm{M}$ : Systemic administration of mesenchymal stem cells increases neuron survival after global cerebral ischemia in vivo (2VO). Neural Plast 2010: 534925, 2010.

23. Ohtaki H, Ylostalo JH, Foraker JE, Robinson AP, Reger RL, Shioda S and Prockop DJ: Stem/progenitor cells from bone marrow decrease neuronal death in global ischemia by modulation of inflammatory/immune responses. Proc Natl Acad Sci USA 105: 14638-14643, 2008.

24. Wang L, Li Y, Chen X, Chen J, Gautam SC, Xu Y and Chopp M: MCP-1, MIP-1, IL-8 and ischemic cerebral tissue enhance human bone marrow stromal cell migration in interface culture. Hematology 7: 113-117, 2002.

25. Zimmer DB, Cornwall EH, Landar A and Song W: The S100 protein family: History, function and expression. Brain Res Bull 37: 417-429, 1995.

26. Wojtczak-Soska K and Lelonek M: S-100B protein: An early prognostic marker after cardiac arrest. Cardiol J 17: 532-536, 2010.

27. Grubb NR, Simpson C, Sherwood RA, Abraha HD, Cobbe SM, O'Carroll RE, Deary I and Fox KA: Prediction of cognitive dysfunction after resuscitation from out-of-hospital cardiac arrest using serum neuron-specific enolase and protein S-100. Heart 93: 1268-1273, 2007. 